\title{
PREVALENCIA DEL HÍGADO GRASO NO ALCOHÓLICO EN ESTUDIANTES DE LA UNIVERSIDAD CATÓLICA DE SANTIAGO DE GUAYAQUIL, PERIODO 2014
} PREVALENCE OF NON-ALCOHOLIC FATTY LIVER IN STUDENTS OF THE CATHOLIC UNIVERSITY OF SANTIAGO DE GUAYAQUIL, PERIOD 2014

\author{
PREVALÊNCIA DE FÍGADO GRASSO NÃO ALCOÓLICO EM ALUNOS DA UNIVERSIDADE CATÓLICA SANTIAGO DE \\ GUAYAQUIL, PERÍODO 2014
}

\author{
DIEGO VÁSQUEZ CEDEÑO', ROBERTO BRIONES JIMÉNEZ', MARTHA CELI MERO', OLGA MUÑOZ ROCA', CARLOS MONCAYO VALENCIA'
}

1 Universidad Católica de Santiago de Guayaquil, Guayaquil, Ecuador

\begin{abstract}
Resumen
Introducción: el hígado graso no alcohólico (HGNA) es una enfermedad inflamatoria del estroma hepático que comprende desde esteatosis hepática hasta cirrosis. Objetivo: identificar la prevalencia de hígado graso no alcohólico estratificado según medidas antropométricas, perfil lipídico y su asociación a los hábitos nutricionales en la Universidad Católica Santiago de Guayaquil. Metodología: se obtuvo una muestra por conveniencia de 285 estudiantes de la Universidad Católica Santiago de Guayaquil. A éstos se le aplicó una encuesta antropométrica, se extrajo muestras de sangre y se realizó ecografía abdominal para determinar los grados de hígado graso no alcohólico. Se analizaron los resultados por medio de la prueba de Kruskal Wallis entre la clasificación ecográfica, medidas antropométricas y los parámetros de laboratorio. Resultados: la prevalencia de hígado graso no alcohólico fue del $37 \%$, los valores antropométricos del índice de masa corporal, perímetros de cadera y cintura estuvieron aumentados significativamente (valor $p=0.001-0.008$ ) en los estudiantes con hígado graso no alcohólico comparado con los que no presentaban este diagnóstico ecográfico. № hubo asociación entre los parámetros bioquímicos elevados y los grados de hígado graso no alcohólico. Conclusión: la prevalencia de hígado graso no alcohólico es alta y semejante a la presentada en la región. Se recomienda fomentar el conocimiento y la prevención del HGNA en los estudiantes universitarios debido a que puede existir una alta cantidad de jóvenes con esta enfermedad sin diagnóstico.
\end{abstract}

PALABRAS CLAVE: hígado graso no alcohólico, ácidos grasos, antropometría, cirrosis hepática.

Abstract

Introduction: non-alcoholic fatty liver disease (NAFLD) is an inflammatory disease of the hepatic stroma that ranges from hepatic steatosis to cirrhosis. Objective: to identify the prevalence of nonalcoholic fatty liver stratified according to anthropometric measurements, lipid profile and its association with nutritional habits at the Santiago de Guayaquil Catholic University. Methodology: a sample was obtained from convenience of 285 students of the Santiago de Guayaquil Catholic University. An anthropometric survey was applied to these patients, blood samples were taken and abdominal ultrasound was performed to determine the degree of nonalcoholic fatty liver. The results were analyzed by means of the Kruskal Wallis test between ultrasound classification, anthropometric measurements and laboratory parameters. Results: the prevalence of nonalcoholic fatty liver was 37\%, the anthropometric values of the body mass index, hip and waist perimeters were significantly increased ( $p$ value $=0.001-0.008$ ) in the students with nonalcoholic fatty liver compared to the ones who did not have an ultrasound diagnosis. There was no association between the elevated biochemical parameters and the degree of non-alcoholic fatty liver. Conclusion: the prevalence of nonalcoholic fatty liver is high and similar to that present in the region. It is recommended to promote knowledge of NAFLD in university students because there may be a high number of young people with this condition undiagnosed.

KEYWORDS: nonalcoholic fatty liver, fatty acids, anthropometry, liver cirrhosis.

Resumo

Introdução: e fígado graso não alcoholico (HGNA) e uma doença inflamatória do estroma hepático que varia da esteatose hepática à cirrose. Objetivo: identificar a prevalência de esteatose hepática não alcoólica estratificada segundo medidas antropométricas, perfil lipídico e sua associação com hábitos alimentares na Universidade Católica de Santiago de Guayaquil. Metodologia: uma amostra foi obtida para a conveniência de 285 estudantes da Universidade Católica de Santiago de Guayaquil. Uma pesquisa antropométrica foi aplicada a esses pacientes, amostras de sangue foram coletadas e ultrassonografia abdominal foi realizada para determinar os graus de fígado grasso não alcoólica. Os resultados foram analisados por meio do teste de Kruskal Wallis entre a classificação ultrassonográfica, as medidas antropométricas e os parâmetros laboratoriais. Resultados: a prevalência de esteatose hepática não alcoólica foi de $37 \%$, os valores antropométricos do índice de massa corporal, perímetros de quadril e cintura aumentaram significativamente (valor $p=0,001-0,008$ ) nos escolares com esteatose hepática não-alcoólica que não tiveram esse diagnóstico ultrassonográfico. Não houve associação entre os parâmetros bioquímicos elevados e os graus de fígado grasso não alcoólico. Conclusão: a prevalência de esteatose hepática não-alcoólica é alta e semelhante à apresentada na região. Recomenda-se promover o conhecimento da DHGNA em estudantes universitários, pois pode haver um número elevado de jovens com essa doença sem diagnóstico.

PALABRAS-CHAVE: fígado gorduroso no alcoólico, ácidos graxos, antropometria, cirrose hepática. 


\section{INTRODUCCIÓN}

El hígado graso no alcohólico (HGNA) es una enfermedad inflamatoria del estroma hepático que comprende desde esteatosis hepática hasta cirrosis e insuficiencia hepática. Esta enfermedad aparece en cualquier edad sin estar relacionada al consumo de alcohol, como antes se sospechaba. La prevalencia del HGNA en adultos oscila entre 10 a $24 \%$ y tiene un riesgo 4 a 6 veces mayor en las personas obesas comparadas con las de normopeso. ${ }^{1}$ La supervivencia a 5 años de pacientes con hígado graso clase III y IV se estima que esta entre un 67 y 59\% respectivamente, aunque la muerte puede acontecer por otras comorbilidades, en especial insuficiencia renal crónica terminal ${ }^{2}$ y de los diagnosticados como HGNA un 20 a $25 \%$ evoluciona a hepatocarcinoma. ${ }^{2,3}$

En Latinoamérica se han realizado algunos estudios de prevalencia del HCNA, que van desde el 17 al 36\% en países como Cuba, Colombia y México. ${ }^{2,4-6}$ En el ámbito local se han descubierto importantes asociaciones entre el perfil lipídico con la presencia de HGNA en pacientes hospitalizados. ${ }^{7,8}$

Teniendo presente este antecedente, centros de masiva afluencia, como la Universidad Católica de Santiago de Guayaquil (UCSC) que es un lugar en el cual los jóvenes, en su mayoría, permanecen estudiando un promedio de 6 horas al día, durante 4 a 5 años mínimo, será interesante demostrar la presencia de esta patología. Esta población susceptible por su alta carga horaria y oferta gastronómica habitual interna, puede desarrollar factores de riesgo nutricionales asociados a hígado graso no alcohólico, que puede pasar desapercibido debido a que es una condición asintomática. ${ }^{7,9,10} \mathrm{El}$ objetivo de este estudio es determinar la frecuencia de HGNA en la población universitaria junto con sus factores de riesgo asociados a esta patología estratificándolo en subgrupos según la severidad del HGNA.2,11-13

\section{MATERIALES Y MÉTODOS}

Se utilizó un diseño transversal donde se evaluaron a los estudiantes de la UCSG. Los criterios de inclusión fueron estudiantes registrados en la universidad por más de 1 año, que permanecieran en el recinto universitario por más de 4 horas al día y consumieran alimentos en la institución. Se excluyeron los estudiantes que no se realizaran los exámenes de laboratorio, la ecografía y la valoración antropométrica completa.
Las variables antropométricas medidas fueron: edad en años, género, facultad en la que estudia (medicina, ingeniería antropométrica), peso en kilogramos $(\mathrm{kg})$, talla en metros, perímetro cintura en centímetros (cm), perímetro de cadera en cm, índice de masa corporal (IMC), y la relación cintura/cadera. Se receptó la presión arterial sistólica y diastólica en $\mathrm{mmHg}$. Se registró los siguientes valores de laboratorio: colesterol total, colesterol de alta densidad (HDL), colesterol de baja densidad (LDL), triglicéridos y glucosa en mg/dl.

Para la valoración antropométrica se llenó una encuesta nutricional que permitió cuantificar el consumo de calorías en 24 horas. Para la evaluación ecográfica se realizó con un ecógrafo MINDRAY ${ }^{\circledR}$ modelo Z6, transductor 3C5P del año 2012, estas ecografías fueron hechas por dos ecografistas del hospital Teodoro Maldonado Carbo en momentos distintos y bajo código alternas para mantener el ciego del operador. Para estadificar el hígado graso no alcohólico se utilizaron los parámetros de Matteoni, el cual lo clasifica en 0 ausente, 1 es leve $<33 \%$, 2 es moderada del 33 al $66 \%$ y 3 severa o intensa cuando es por encima del $66 \%$ de afectación del parénquima hepático por evaluación ecográfica.

De los 14125 alumnos inscritos durante el periodo 2014, se realizó un cálculo de muestra para distribución normal asumiendo un universo de 14.125 estudiantes, para un alfa de 0,01 , con intervalo de confianza del $99 \%$ y distribución de respuestas de 0,5; dió un tamaño de la muestra de 180 estudiantes. Se realizó un análisis estadístico descriptivo de frecuencias y porcentajes para variables cualitativas y media con desviación estándar para las variables cuantitativas verificando si su distribución era normal, caso contrario se utiliza mediana y rango intercuartílico. Se determinó si una variable cuantitativa tenía una distribución normal mediante la prueba de Shapiro Wilk. Se realizó análisis de estadística inferencial contrastando sus valores por la prueba de Chi2 y Kruskal Wallis según fuera apropiado. Posteriormente utilizando la correlación de Spearman, se buscó asociación entre HGNA con las variables antropométricas y bioquímicas mediante un análisis bivariado entre la presencia y ausencia de hígado graso. Todos estos cálculos se realizaron en Microsoft Excel $^{\circledR}$ para Windows 2013 y STATA ${ }^{\circledR}$ versión 12 para Windows. Se consideró los valores estadísticamente significativos si presentaban un valor p menor de 0,05. Para la realización de este estudio se siguieron las recomendaciones de 
la Declaración de Helsinki. Todos los estudiantes que participaron firmaron un consentimiento informado.

\section{RESULTADOS}

En la revisión, de los 180 voluntarios, 170 se realizaron todos los exámenes, negaron el consumo de alcohol y tenían las valoraciones ecográficas establecidas para este estudio. La distribución de las variables fue no paramétrica para las variables numéricas mediante la prueba de Shapiro Wilk, excepto de la edad $\mathrm{p}=0.02$ por lo que se utilizó las medianas y rangos intercuartílicos en las variables cuantitativas. La frecuencia de HGNA fue del 37\% IC 95\% (33.3-47.36), (Figura 1).

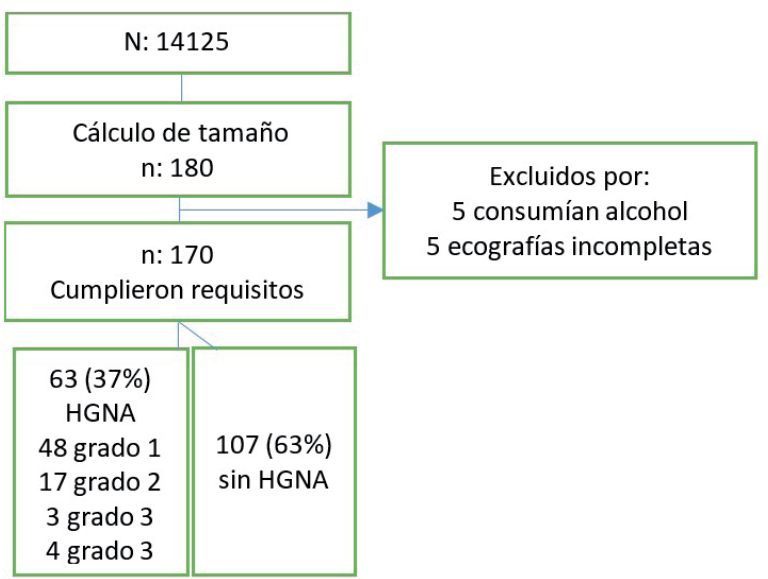

Figura 1. Flujograma.

De los estudiantes con HGNA, 55 (51\%) fueron de genero masculino, además se observó que la edad aumentaba progresivamente con los grados de HCNA siendo esta asociación estadísticamente significativa (valor $\mathrm{p}=<0.001$ ) (figura 2) y los estudiantes de la carrera de medicina presentaron mayor cantidad de casos con un $76 \%$ (valor $\mathrm{p}=0.03)$, (Tabla 1 ).

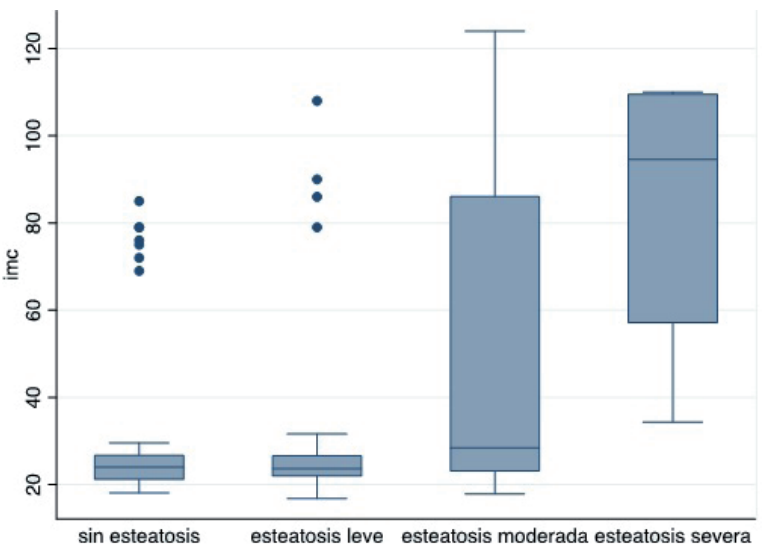

Figura 2. Relación entre la edad y esteatosis
TABLA 1. GRADOS DE HGNA POR VARIABLE.

\begin{tabular}{|c|c|c|c|c|c|}
\hline VARIABLES & $\begin{array}{l}\text { NO HGNA } \\
\text { N: } 119 \\
(60 \%)\end{array}$ & $\begin{array}{l}\text { GRADO } 1 \\
\text { N: } 55 \\
(28 \%)\end{array}$ & $\begin{array}{l}\text { GRADO } 2 \\
\text { N: } 21 \\
(10 \%)\end{array}$ & $\begin{array}{l}\text { GRADO } 3 \\
N: 4 \\
(2 \%)\end{array}$ & $\begin{array}{l}\text { VALOR P } \\
<0.05\end{array}$ \\
\hline $\mathrm{xO \dagger}$ & $\begin{array}{r}\text { F: } 27(48 \%) \\
\text { M: } 29 \\
\text { (51\%) }\end{array}$ & $\begin{array}{l}\text { F: } 25(66 \%) \\
\text { M: } 3(34 \%)\end{array}$ & $\begin{array}{l}\text { F: } 6(55 \%) \\
\text { M: } 5(45 \%)\end{array}$ & $\begin{array}{l}\text { H: } 3(75 \%) \\
\text { F: } 1(25 \%)\end{array}$ & 0.487 \\
\hline \multicolumn{6}{|c|}{ Mediana y Rango Intercuartílico } \\
\hline Edad‡ & $19( \pm 2)$ & $20( \pm 4)$ & $22( \pm 13)$ & 23() & $<0.001^{* *}$ \\
\hline Facultad & $\begin{array}{l}\text { Medicina } \\
46(76 \%)\end{array}$ & $\begin{array}{r}\text { Ingeniería } \\
23(41 \%)\end{array}$ & $\begin{array}{r}\text { Medicina } 13 \\
(61 \%)\end{array}$ & $\begin{array}{r}\text { Medicina } \\
3(75 \%)\end{array}$ & $0.03^{*}$ \\
\hline IMCF & $24( \pm 5)$ & $23( \pm 5)$ & 26. $( \pm 8)$ & 34 & $0.021^{*}$ \\
\hline PAS‡ & $\begin{array}{r}113.93 \\
( \pm 10) \\
\end{array}$ & $111.94( \pm 12)$ & $109.6( \pm 20)$ & 110 & 0.654 \\
\hline PAD‡ & $.87( \pm 10)$ & $72.91( \pm 10)$ & $72.9( \pm 15)$ & 70 & 0.950 \\
\hline P. Caderał & $97.41( \pm 10)$ & $99.96( \pm 8)$ & $101.75( \pm 8)$ & 120 & $0.008^{*}$ \\
\hline P. Cinturał & $80.26( \pm 12)$ & $80.61( \pm 12)$ & $84.6( \pm 20)$ & 119 & $0.001^{* *}$ \\
\hline $\begin{array}{l}\text { Calorías } \\
24 h \neq\end{array}$ & $\begin{array}{r}1227 \\
( \pm 992) \\
\end{array}$ & $1308( \pm 995)$ & $1154( \pm 816)$ & 2315 & 0.843 \\
\hline LDLf & $85( \pm 17)$ & $90.17( \pm 30)$ & $93.73( \pm 44)$ & 130 & 0.279 \\
\hline HDLf & $47,16( \pm 10)$ & $47,02( \pm 15)$ & $47,73( \pm 13)$ & 33.6 & 0.730 \\
\hline $\begin{array}{l}\text { Triglicéri- } \\
\text { dosł }\end{array}$ & $75,46( \pm 21)$ & $73.40( \pm 45)$ & $87,62( \pm 95)$ & 227 & 0.032 \\
\hline & & & & & \\
\hline
\end{tabular}

$\mathrm{F}=$ femenino, $\mathrm{M}=$ masculino, $\quad I M C=$ índice de masa corporal, $\mathrm{PAS}=$ presión arterial sistólica, $\mathrm{PAD}=$ presión arterial diastólica, $\mathrm{LDL}=$ colesterol de baja densidad, $\mathrm{HDL}=$ colesterol de alta densidad. $\dagger=$ frecuencia (porcentaje), $\neq=$ media ( \pm desviación estándar), ${ }^{*}$ Diferencia significativa, ${ }^{* *}$ Diferencia muy significativa.

En la figura 3 se observa que los grados de HCNA eran más severos conforme aumentaba el IMC esta asociación fue estadísticamente significativa (valor $\mathrm{p}=0.021$ ) (Tabla 1). No se encontró asociación estadísticamente significativa entre la presiones sistólicas y diastólicas con los niveles de HGNA (valor $\mathrm{p}=0.654$ y valor $\mathrm{p}=0.950$ respectivamente), (Tabla 1 ).

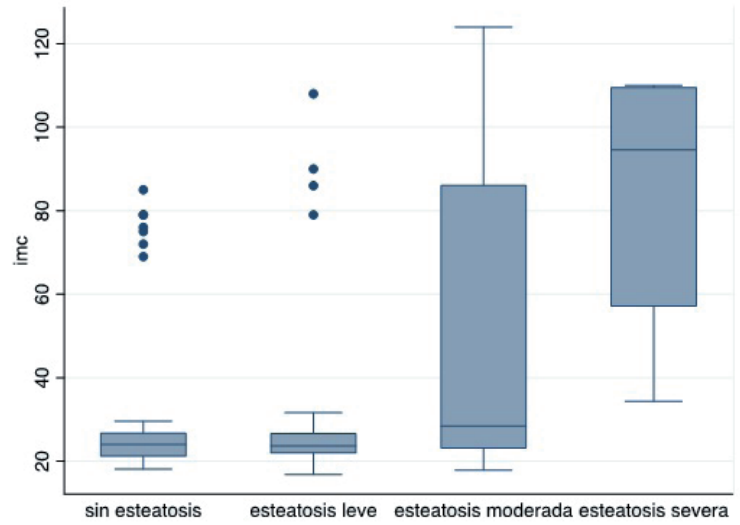

Figura 3. Relación IMC y esteatosis

En los valores bioquímicos se observó que la glucosa (valor $\mathrm{p}=0.998$ ), HDL (valor $\mathrm{p}=0.730$ ) y LDL (valor $\mathrm{p}=0.279$ ) colesterol no se aumentaron con los diferentes niveles de HGNA ubicándose en rangos normales; en cambio los niveles de 
triglicéridos si se elevaron significativamente (valor $\mathrm{p}=0.032$ ), pero también se mantenían dentro de los rangos normales. Además el consumo de calorías en 24 horas no tuvo una asociación estadísticamente significativa con los niveles de HGNA (valor $\mathrm{p}=0.84$ ), (Tabla 1 ). Al utilizar la correlación de Spearman se encontró una asociación estadísticamente significativa con el IMC y perímetro de cadera (valor $\mathrm{p}=<0,001$ y 0,004 respectivamente). No se encontró diferencia estadísticamente significativa al valorar perímetro de cintura, consumo de proteínas en 24 horas ni con los triglicéridos, (Tabla 2).

\begin{tabular}{|c|c|c|}
\hline CORRELACIÓN & $\mathbf{R}$ & VALOR P \\
\hline HGNA e IMC & 0.351 & $<0.001$ \\
\hline HGNA y perímetro de cintura & 0.247 & 0.20 \\
\hline HGNA y perímetro de cadera & 0.318 & 0.004 \\
\hline HGNA y calorías en 24 horas & 0.024 & 0.854 \\
\hline HGNA y triglicéridos & 0.060 & 0.641 \\
\hline
\end{tabular}

DISCUSIÓN

La frecuencia del hígado graso no alcohólico fue de un $37 \%$ en esta muestra; datos similares se han encontrado en otras poblaciones adolecentes con obesidad en países como México; sin embargo, la mayoría de nuestra población es adulta y con normopeso. Es de gran importancia esta presencia de HGNA ya que su asociación a eventos cardiovasculares expone a un riesgo elevado a los estudiantes universitarios. ${ }^{4,5,10}$

El IMC fue una de las variables con mayor asociación estadística con el HCNA en adultos. Sin embargo las variables bioquímicas como HDL, LDL, glucosa y triglicéridos, se encontraban dentro de los parámetros normales, debido a que la edad de los participantes fluctuaba entre los 19 a 24 años y aún los mecanismos homeostáticos regulan los niveles de estas sustancias. ${ }^{14,15}$ En contraposición con pacientes de edades superiores, donde sí hubo alteraciones bioquímicas relacionadas al aumento del IMC. ${ }^{7}$ Existen teorías por las cuales las personas menores de 30 años no presenten alteraciones bioquímicas del sobrepeso u obesidad, una de ellas es que el IGF1 en este grupo de personas aún circula en niveles normales evitando que la insulina circulante se quede fuera del medio extracelular, lo cual permite el buen metabolismo de los carbohidratos y de las grasas. Otras teorías se basan en que la lecitina ejerce efecto en el metabolismo de los ácidos grasos, también actúa en estos grupos poblacionales, lo cual pierde su efecto en grupos de mayor edad. ${ }^{16,17}$

El motivo por el que no se pudo recolectar adecuadamente todos los datos de los pacientes fue a las dificultades que los estudiantes presentaron para realizarse los exámenes de laboratorio y la ecografía; ésta fue la causa del 5,5\% de la pérdida de la muestra. Además la muestra constó de una mayor cantidad de estudiantes de la facultad de ciencias médicas, lo que le quita generabilidad a los resultados al aplicarlos a toda la universidad.

\section{CONCLUSIONES Y RECOMENDACIONES}

La frecuencia de HGNA en estudiantes de la Universidad Católica Santiago de Guayaquil es elevada y es importante seguir realizando estudios con poblaciones de mayor tamaño y más representativas. En pacientes jóvenes con valores antropométricos y bioquímicos dentro de parámetros normales, parece ser que la asociación más importante de la HGNA es con el IMC. Basados en estos resultados se recomienda fomentar la información sobre HGNA en los estudiantes universitarios, debido a que puede existir una alta cantidad de jóvenes con esta enfermedad y sin diagnóstico.

\section{AGRADECIMIENTOS}

Se deja constancia del agradecimiento a los colaboradores del presente trabajo, a la Universidad Católica de Santiago de Guayaquil por permitir realizar este estudio de gran importancia para la comunidad universitaria; además a los alumnos del 4 y 9 ciclo de la carrera de medicina y nutrición clínica, por su asesoramiento en las valoraciones nutricionales; también al ecografista Dr. Andrés Sanango, por su contribución trascendental en el mismo.

\section{REFERENCIAS BIBLIOGRÁFICAS}

1. Musso G, Gambino R, De Michieli F, Cassader M, Rizzetto M, Durazzo M, et al. Dietary habits and their relations to insulin resistance and postprandial lipemia in nonalcoholic steatohepatitis. Hepatology. 2003;37(4):909-16.

2. Santos LF, Hernández G, Varón Puerta A, Beltrán Ó, Botero RC, Mejía G. Non alcoholic fatty liver disease: The new millennium pandemia. Rev Colomb Gastroenterol. diciembre de 2010;25(4):380-98.

3. Mozumdar A, Liguori G. Persistent Increase of Prevalence of Metabolic Syndrome Among U.S. Adults: 
NHANES III to NHANES 1999-2006. Diabetes Care. 1 de enero de 2011;34(1):216-9.

4. JL-C, Di L, Nc C-T, Me O, Mu E. Prevalencia de higado graso no alcohólico y síndrome metabólico en población asintomática. Rev Gastroenterol Mx. 2006; 71(4): 453-9.

5. Pérez Lorenzo M, Duarte Castillo N, Montero González T, Franco Estrada S, Winograd Lay R, Brizuela Quintanilla RA. Prevalencia del hígado graso no alcohólico en muestras de biopsias hepáticas. Rev Cuba Med Mil. diciembre de 2006;35(4):0-0.

6. Vernon G, Baranova A, Younossi ZM. Systematic review: the epidemiology and natural history of non-alcoholic fatty liver disease and non-alcoholic steatohepatitis in adults. Aliment Pharmacol Ther. 1 de agosto de 2011;34(3):274-85.

7. Vásquez-Cedeño D, Guerrero-Arellano L, Torre FL-D la. Correlación de la incidencia de esteatosis hepática no alcohólica de diagnóstico ecográfico con medidas antropométricas y parámetros bioquímicos en el Hospital Luis Vernaza, periodo de abril a julio 2013. Rev MedPre. 23 de marzo de 2014;4(1):31-6.

8. Vásquez-Cedeño DA, Tamariz Amador E, Cevallos Abad MI. Lipid profile in patients with newly diagnosed coronary heart disease: 2012 and 2013 crosssectional study in Luis Vernaza Hospital, Ecuador. Medwave. 14 de agosto de 2014;14(07):e6007-e6007.

9. Factores de riesgo asociados a la presencia de hígado graso no alcohólico: un estudio de casos y controles [Internet]. [citado 12 de noviembre de 2014]. Disponible en: http://www.sciencedirect.com/science/ article/pii/S0025775313001905

10. Chao J, Huo T-I, Cheng H-Y, Tsai J-C, Liao J-W, Lee M-S, et al. Gallic Acid Ameliorated Impaired Glucose and Lipid Homeostasis in High Fat Diet-Induced NAFLD Mice. PLoS ONE. 11 de junio de 2014;9(6):e96969.

11. Bantel H, John K, Schulze-Osthoff K. Robust detection of liver steatosis and staging of NAFLD by an improved
ELISA for serum cytokeratin-18 fragments. Am J Gastroenterol. enero de 2014;109(1):140-1.

12. Correa-Carhuachin KV, Manayalle-Torres CM, León-Jiménez FE, Díaz-Vélez C, Cubas-Benavides F. Conocimiento sobre hígado graso no alcohólico en médicos generales de dos distritos de Chiclayo, Perú, durante el 2012. Rev Gastroenterol Perú. enero de 2014;34(1):29-32.

13. López-Capapé M, López-Bermejo A, Alonso Blanco M, Lara Orejas E, Corbatón Blasco J, Barrio Castellanos R. Esteatosis hepática, resistencia a la insulina y adiponectina en una población con obesidad. An Pediatría. diciembre de 2009;71(6):495-501.

14. González Jiménez E, Río-Valle JS, Ferre JÁ. Esteatosis hepática y su manejo clínico en el adolescente obeso. Endocrinol Nutr. enero de 2011;58(1):32-7.

15. Santomauro M, Paoli-Valeri M, Fernández M, Camacho N, Molina Z, Cicchetti R, et al. Hígado graso no alcohólico y su asociación con variables clínicas y bioquímicas en niños y adolescentes obesos: efecto de un año de intervención en el estilo de vida. Endocrinol Nutr. junio de 2012;59(6):346-53.

16. Palenzuela DL, Calvo MG, Avero P. EJERCICIO FÍsICO REGULAR COMO UN MECANISMO DE PROTECCIÓN CONTRA LA DEPRESIÓN EN JÓVENES. Psicothema. 31 de diciembre de 1998;10(Número 1):29-39.

17. Vásquez-Cedeño D, Guerrero-Arellano L. Correlación de la incidencia de esteatosis hepática no alcohólica de diagnóstico ecográfico con medidas antropométricas y parámetros bioquímicos en el Hospital Luis Vernaza, periodo de abril a julio 2013 | Vásquez-Cedeño | Revista MedPre. ISSN 1390-8456 [Internet]. Correlación de la incidencia de esteatosis hepática no alcohólica de diagnóstico ecográfico con medidas antropométricas y parámetros bioquímicos en el Hospital Luis Vernaza, periodo de abril a julio 2013. [citado 15 de noviembre de 2014]. Disponible en: http://medpre. med.ec/ojs/index.php/revistamedpre/article/view/79. 